\title{
Comparison of acoustic features of word accent in English and Japanese
}

\author{
Hiroya Fujisaki,* Keikichi Hirose,* and Miyoko Sugito** \\ *Faculty of Engineering, University of Tokyo, \\ 7-3-1, Hongo, Bunkyo-ku, Tokyo, 113 Japan \\ **Faculty of Liberal Arts, Osaka Shoin Women's College, \\ 4-2-26, Hishiyanishi, Higashiosaka, 577 Japan
}

(Received 11 April 1985)

\begin{abstract}
In order to determine the universal and language-specific characteristics for word accent, its acoustic manifestations were analyzed and compared in the disyllabic words of English ("permit," "record," "object" etc.) and in the two-mora words of Japanese ("ame"). Analyses on the fundamental frequency contours ( $F_{0}$ contours) of these words were made using the functional model of $F_{0}$ contour generation proposed by one of the authors. While a marked similarity was observed between $F_{0}$ contour characteristics of English and Japanese in cases of both first-syllable accented and second-syllable accented, individual differences were much greater in the accent command for English words with an accented first syllable. Segmental and syllabic durations were measured on the speech waveform, and it was found that the accentual changes in duration occur mainly in the second syllable in Japanese, while in English they tend to be complementary in the first and the second syllables. The intensity and formant frequencies of syllabic nuclei were also analyzed. The results of these analyses indicated that duration, intensity and formant frequencies are less stable than $F_{0}$ contour as correlates for the word accent both in English and in Japanese.
\end{abstract}

PACS number: $43.70 . \mathrm{Gr}$

\section{INTRODUCTION}

Word accent plays an important role in speech communication, but its acoustic features usually differ from language to language. As such acoustic features we can note fundamental frequency, intensity, segmental duration, formant frequencies, and others. It is well known that the voice fundamental frequency is the most important feature of word accent in Japanese, but the segmental duration is also known to be important in English. ${ }^{1}$ While perception of stress in English has been extensively studied mainly using synthetic speech sounds, ${ }^{2)}$ and an attempt has also been made to simulate human judgment of stress in connected speech by a digital computer, ${ }^{3)}$ we still seem to need detailed analysis of the acoustic characteristics. We have already analyzed the fundamental frequency contour (henceforth $F_{0}$ contour) of two-mora Japanese words in the Tokyo and the Osaka dialects, and our findings have revealed the relation between its pattern and accent type. $^{4,5)}$ In this paper the fundamental frequency, intensity, segmental duration, and formant frequencies of disyllabic English words will be analyzed and compared with those of two-mora Japanese words, in order to determine both universal and language-specific characteristics of word accent. ${ }^{6)}$

\section{SPEECH MATERIALS}

The English words chosen for the present analysis were "permit," "record," "object," and others in which a change of part of speech from noun to verb is associated with a change in accent position from the first to the second syllable. Four native speakers, 
The symbols in Eq. (1) to Eq. (3) indicate

$F_{\text {min }}$ : asymptotic value of $F_{0}$ in the absence of accent component,

$A_{\mathrm{u} 1}$ : magnitude of the positive utterance command,

$A_{\mathrm{u} 2}:$ magnitude of the negative utterance command,

$A_{\mathrm{a}}:$ amplitude of the accent command,

$T_{0}$ : timing of the positive utterance command,

$T_{3}$ : timing of the negative utterance command,

$T_{1}$ : onset of the accent command,

$T_{2}$ : end of the accent command, $\alpha:$ natural angular frequency of the utterance control mechanism,

$\beta$ : natural angular frequency of the accent control mechanism.

The term $A_{\mathrm{u} 2} G_{\mathrm{u}}\left(t-T_{3}\right)$ in Eq. (1) corresponds to a rapid downfall in the baseline, often observed at the end of utterance, and $A_{\mathrm{u} 2}$ has a negative value. In the present analysis, $A_{\mathrm{u} 2}$ was assumed to be equal to $-A_{\mathrm{u} 1}$.

\subsection{Result of Analysis}

The fundamental frequencies were extracted at 10
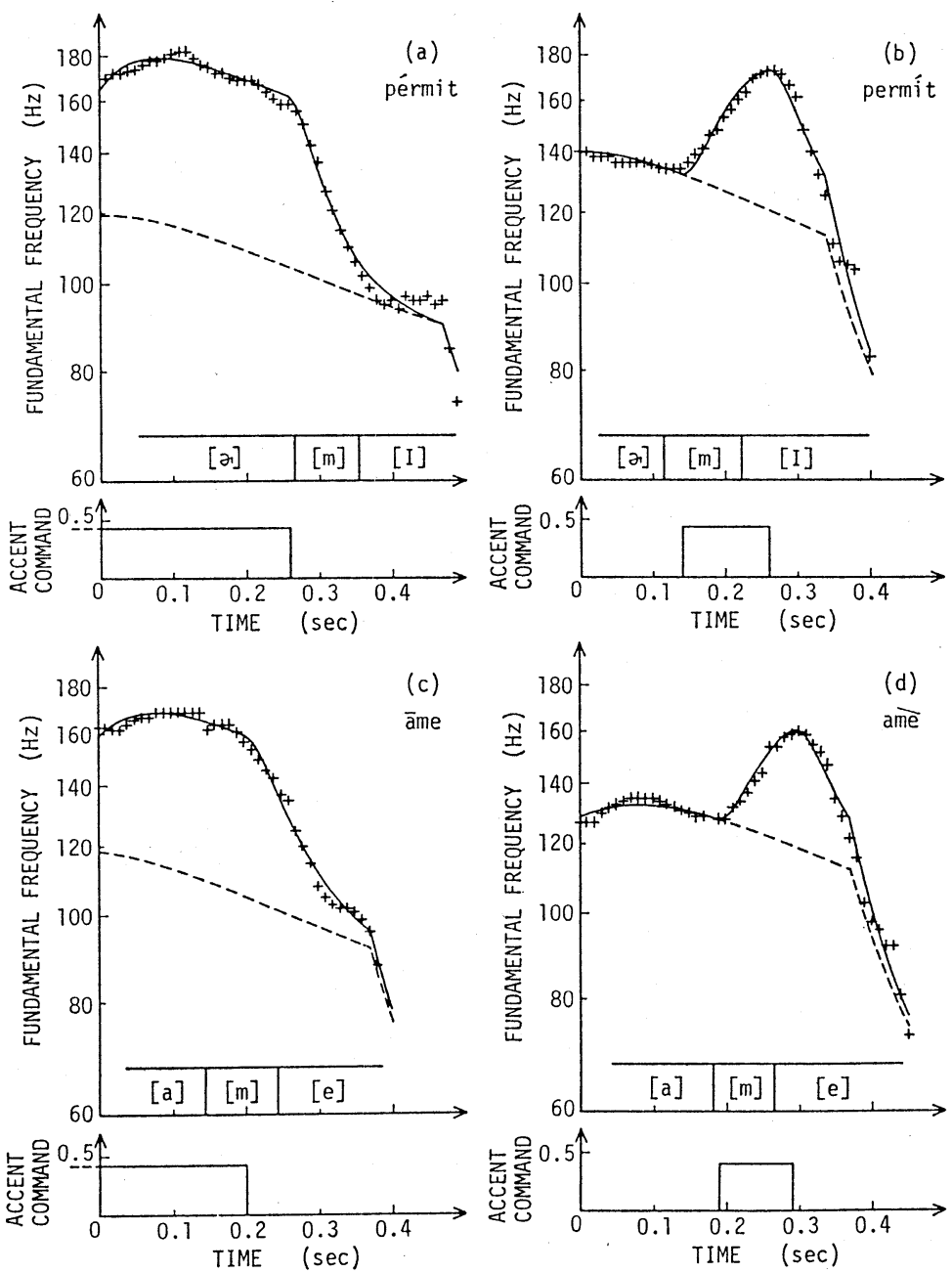

Fig. 2 Analysis-by-synthesis of the $F_{0}$ contours in (a) "pérmit" and in (b) "permít" by informant TB, also with those in (c) "āme" and in (d) "ame" by the Japanese informant ST. The dashed line indicates the utterance component, while the stepwise waveform indicates the timing and the amplitude of the accent command. 
The symbols in Eq. (1) to Eq. (3) indicate

$F_{\text {min }}$ : asymptotic value of $F_{0}$ in the absence of accent component,

$A_{\mathrm{u} 1}$ : magnitude of the positive utterance command,

$A_{\mathrm{u} 2}:$ magnitude of the negative utterance command,

$A_{\mathrm{a}}:$ amplitude of the accent command,

$T_{0}$ : timing of the positive utterance command,

$T_{3}$ : timing of the negative utterance command,

$T_{1}$ : onset of the accent command,

$T_{2}$ : end of the accent command, $\alpha:$ natural angular frequency of the utterance control mechanism,

$\beta$ : natural angular frequency of the accent control mechanism.

The term $A_{\mathrm{u} 2} G_{\mathrm{u}}\left(t-T_{3}\right)$ in Eq. (1) corresponds to a rapid downfall in the baseline, often observed at the end of utterance, and $A_{\mathrm{u} 2}$ has a negative value. In the present analysis, $A_{\mathrm{u} 2}$ was assumed to be equal to $-A_{\mathrm{u} 1}$.

\subsection{Result of Analysis}

The fundamental frequencies were extracted at 10
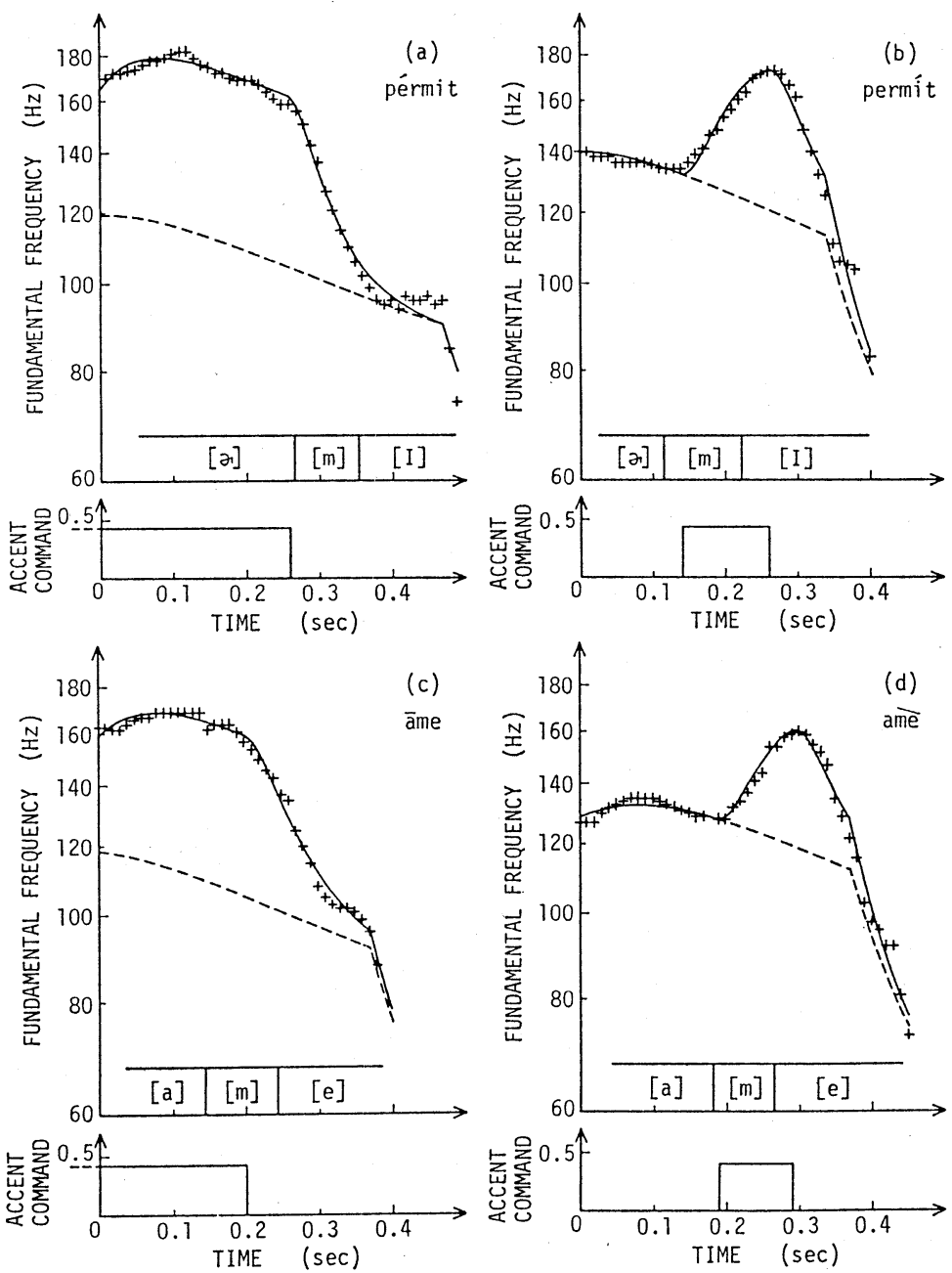

Fig. 2 Analysis-by-synthesis of the $F_{0}$ contours in (a) "pérmit" and in (b) "permít" by informant TB, also with those in (c) "āme" and in (d) "ame" by the Japanese informant ST. The dashed line indicates the utterance component, while the stepwise waveform indicates the timing and the amplitude of the accent command. 
ms (100 sampling points) steps using a short-time autocorrelation analysis of the residual signal from an LPC analysis. The order of the analysis is 11 and the window length is $20 \mathrm{~ms}$. The parameters of the above-mentioned model were determined by minimizing the mean squared error between the extracted $F_{0}$ contour and that of the model on the logarithmic scale. The accentual features are well represented by the onset $T_{1}$ and offset $T_{2}$ of the accent command.

Examples of the extracted fundamental frequencies (indicated by " + ") and the best approximations by the model (solid curves) are shown in Fig. 2 for the voiced segments of (a) "pérmit" and (b) "permít" by informant TB. Fundamental frequency contours similar to these contours for "permit" were observed for Japanese words "āme" and "ame" as shown in Fig. 2 (c) and (d). It is appropriate, therefore, to compare English words of firstsyllable accented with "āme" and those of secondsyllable accented with "ame" for the following discussions. The stepwise waveforms in the figure schematically indicate the accent commands. The end of the accent command for "pérmit" in (a) lags behind the voice onset by about $260 \mathrm{~ms}$, producing a marked similarity to the $F_{0}$ contour of "āme" in (c), but it was found to be located much closer to the voice onset in the other informants of English. The individual differences in the $F_{0}$ contours for "permit were rather small as compared with those for "pérmit."

The onset and end of the accent command for "permit" and "content" are shown in Fig. 3 (a) for informant TB. These values were normalized using the value for the timing of the negative utterance command $T_{3}$ in order to remove the effect of durational variations in each utterance. The characteristics of the accent timing for "permit" were similar to those for "content." This similarity was also found with the other informants of English. The timing of the accent command for "ame" by the Japanese informant ST is shown in Fig. 3 (b), indicating characteristics quite similar to those for the English words. From these results, it may be concluded that word accent types can be well classified by the timing of the accent command both in English and in Japanese.

\section{INTENSITY OF SYLLABIC NUCLEI}

The intensity of a speech waveform reflects not only the intensity of the source but also the effects of the vocal tract transfer function and varies with vowel formant frequencies. In the present study, however, we simply selected an interval of a few fundamental periods from the waveform of each syllabic nucleus where the amplitude appeared to be maximum, and represented the intensity of the syllabic nucleus by the root mean squared amplitude over the selected interval. The intensity ratio $I_{1} / I_{2}$ (in $\mathrm{dB}$ ) of the first to the second syllabic nucleus was adopted as the measure for the effect of accent position on the intensity pattern of a word.

Figure 4 compares the results for English "permit," "content," and "record" by informant TB with
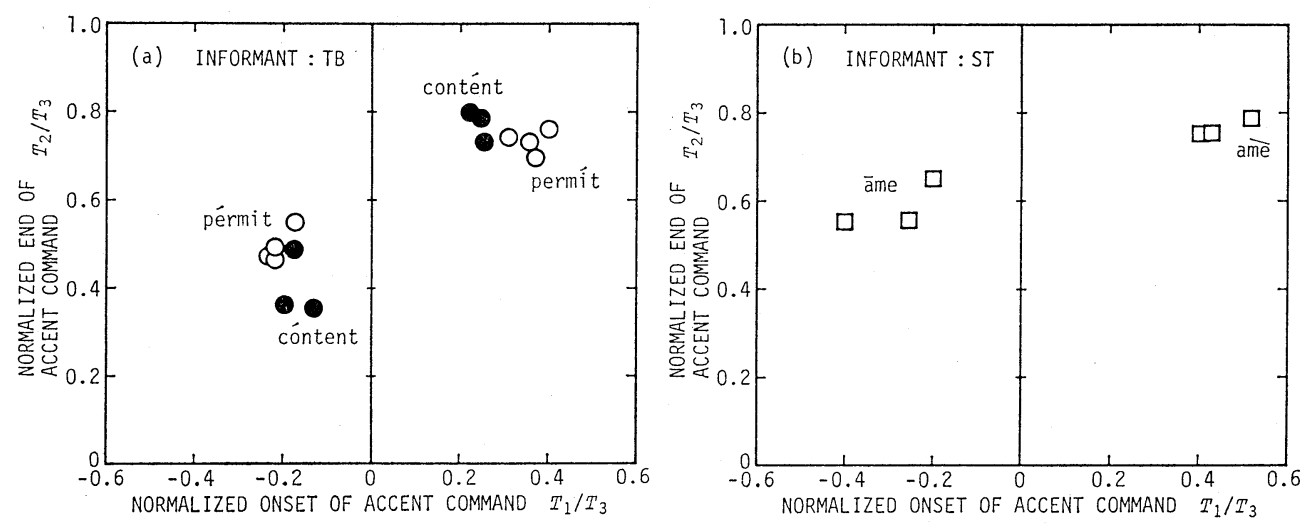

Fig. 3 Accentual changes in the timing of the accent command for (a) "permit" (symbol $\bigcirc$ ), and "content" (symbol $\bullet$ ) by informant TB, and (b) "ame" by the Japanese informant ST. 


\section{H. FUJISAKI et al: : WORD ACCENT FEATURES OF ENGLISH AND JAPANESE}

those for Japanese "ame" by informant ST. The abscissa of the figure is the fundamental frequency ratio $F_{01} / F_{02}$ (in $\mathrm{dB}$ ) of the first to the second syllabic nucleus. The intensity ratio is affected by the change in fundamental frequency. This relationship can be represented roughly by the dashed line in the figure. Therefore, the virtical displacement of each observed point from the dashed line indicates the approximate intensity ratio free from the effect of the

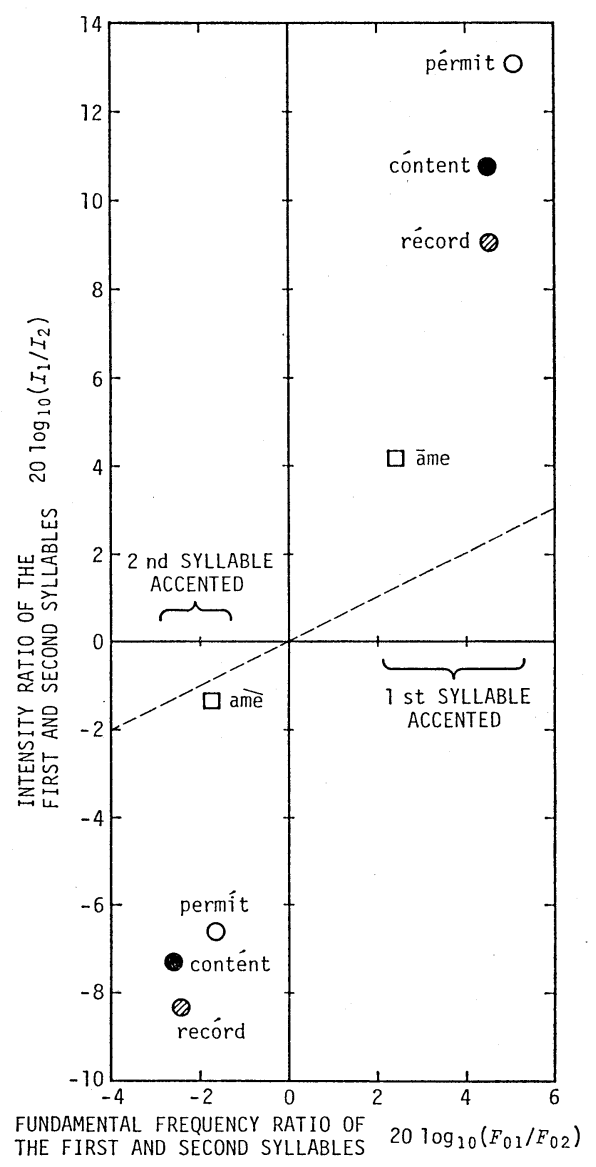

Fig. 4 Intensity ratios of the first to the second syllabic nuclei versus their fundamental frequency ratios for "permit" (symbol $\bigcirc$ ), "content" (symbol @), "record" (symbol ), and "ame" (symbol $\square$ ). Each symbol shows the result averaged over several utterance samples. The informant for the English words was TB. The intensity of the first and second syllables are respectively denoted by $I_{1}$ and $I_{2}$, while the fundamental frequency of those are denoted by $F_{01}$ and $F_{02}$. fundamental frequency change. It can be seen that the effect of accent position in the intensity ratio is much greater in English than in Japanese. The results obtained for the other informants also show the same tendency but indicate considerable individual differences in the magnitude of the intensity ratio.

\section{SEGMENTAL AND SYLLABIC DURATIONS}

In order to analyze the accentual changes in duration, the speech waveform was divided into phonetic segments. For instance, "permit" was segmented on the basis of the following definitions:

[p]: from the noise burst to the voice onset,

$[x]$ : from the voice onset to the onset of oral occlusion,

[m]: from the onset to the offset of oral occlusion,

[I]: from the offset of oral occlusion to the voice offset,

$\varnothing$ : from the voice offset to the noise burst (stop gap),

[t]: from the noise burst to the end of aspiration.

The duration of these segments was measured directly from the waveform by referring to the sound spectrogram, and was averaged over several utterance samples. Figure 5 shows the segmental duration for "permit" by informant TB and those for "ame" by the Japanese informant ST. The segment [t] of

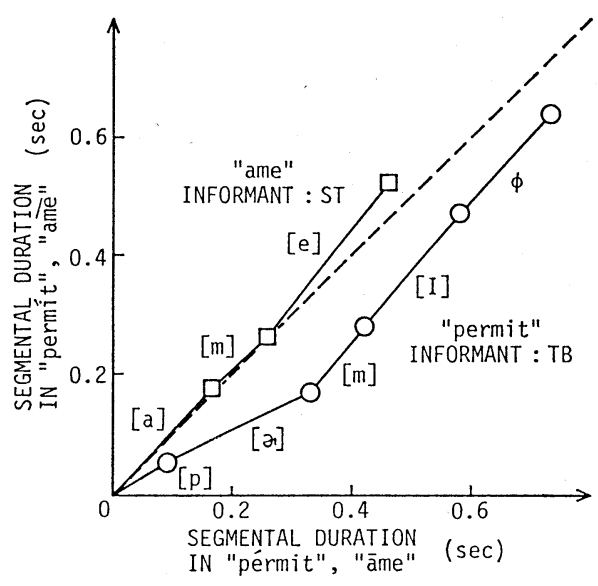

Fig. 5 Comparison of segmental durations in the two accentual types of "permit" by informant TB, and "ame" by the Japanese informant ST. 
"permit" is not shown in the figure, since the end of aspiration was not well defined and varied greatly from utterance to utterance. The solid line indicates the relationship between the cumulative durations of "pérmit," "āme" (abscissa) and "permít," "ame", (ordinate). The deviation of the solid line from the dashed line indicates the change in segmental durations due to accentual change. The duration of [p] and $\left[{ }^{\prime}\right]$ are larger in "pérmit" than in "permít,"

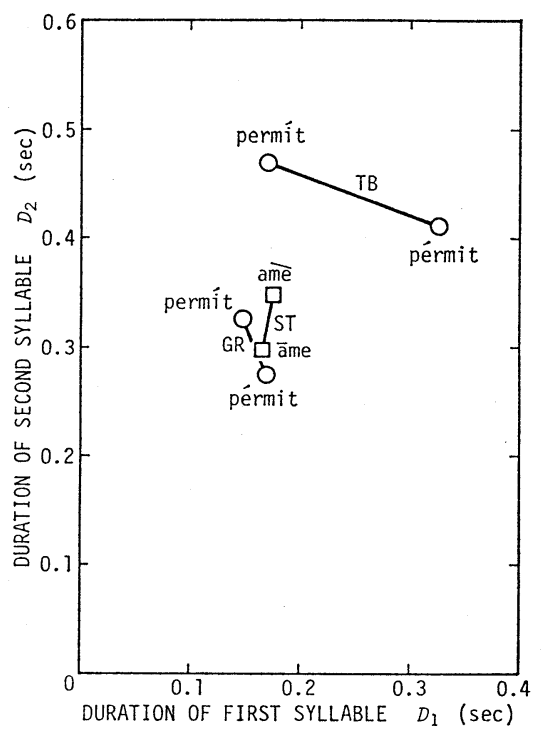

Fig. 6 Change in syllabic durations brought about by accentual change in "permit" by informant TB and GR, and "ame" by the Japanese informant ST. while the reverse is true for [m], [I] and ø. Thus it may be appropriate to define the first syllable as consisting of [p] and [ə]], and the second syllable as consisting of the rest. On the other hand, the change of accentual position in "ame" has hardly any effect at all upon the duration of [a] and [m] but affects the duration of the final [e]. Following the traditional difinition, however, we will define the first mora as consisting of [a], and the second mora as consisting of $[\mathrm{m}]$ and [e].

The changes in the duration of the syllable (or morae) brought about by changes in accentual position are shown in Fig. 6. The final segment [t] was excluded in measuring the duration of the second syllable of "permit." In "ame," accentual changes were only found in the duration of the second mora, while in "permit" complementary changes were found in the first and the second syllables.

\section{FORMANT FREQUENCIES OF SYLLABIC NUCLEI}

Formant frequencies were extracted pitch-synchronously from the short-time frequency spectra of the speech data, ${ }^{8)}$ and the points at which their trajectories were most stationary were selected to determine the formant frequencies of the syllabic nuclei. Panels 7 (a) and (b) in Fig. 7 respectively show the results for "pérmit/permít" and "récord/recórd." While the accentual changes in the formant frequencies are rather small in "pérmit/permít" as compared to "récord/recórd." However, the accentual changes in the formant frequencies for "ame" were found to be much smaller than those for "permit."
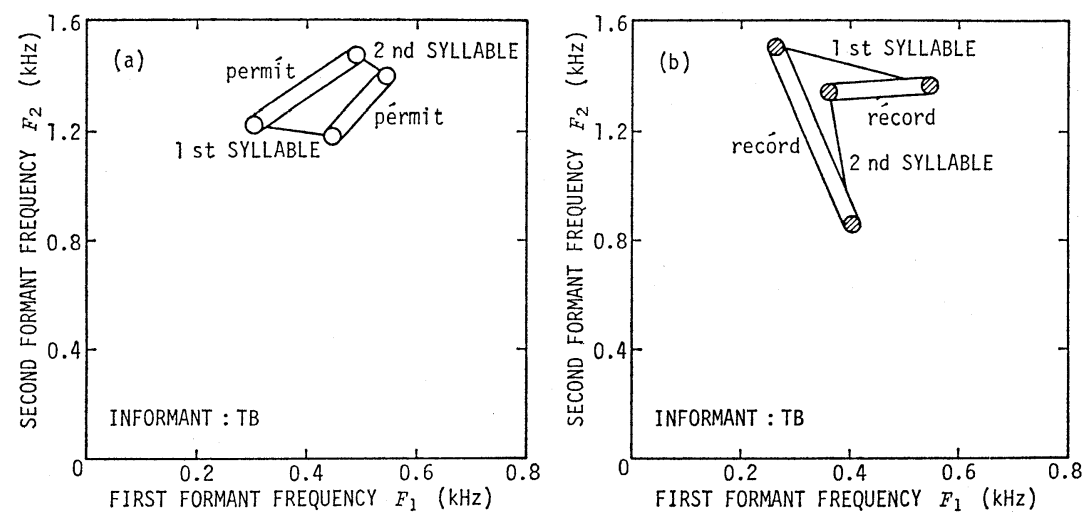

Fig. 7 Accentual changes in the first and second formant frequencies for (a) "permit" and (b) "record" by informant TB. The results were averaged over several utterance samples. 


\section{H. FUJISAKI et al.: WORD ACCENT FEATURES OF ENGLISH AND JAPANESE}

\section{SUMMARY AND CONCLUSION}

For the purpose of distinguishing universal from language-specific correlates of word accent, several samples of disyllabic English words and two-mora Japanese words, uttered by several informants, were analyzed and the effects of accent position on the fundamental frequency contour, intensity of the syllabic nuclei, segmental and syllabic duration, and vowel formant frequencies were investigated. The results indicated that the fundamental frequency contour plays an important role in the transmission of the accentual information both in English and in Japanese. Other correlates, namely intensity, duration and formant frequency, also serve to transmit the accentual information in English, but display greater variability due to word and individual differences.

The foregoing results have been obtained from the analysis of a small number of word samples uttered in isolation by several informants. Their generality needs to be confirmed by further investigation of the effects of phonemic composition, number of syllables, individual differences, and context.

\section{REFERENCES}

1) D. B. Fry, "Duration and intensity as physical correlates of linguistic stress," J. Acoust. Soc. Am. 27, 765-768 (1955).

2) D. B. Fry, "Experiments in the perception of stress," Lang. Speech 1, 126-152 (1958).

3) J. Y. Cheung, A. D. C. Holden, and F. D. Minifie, "Computer recognition of linguistic stress patterns in connected speech," IEEE Trans. ASSP-25, 252256 (1977).

4) H. Fujisaki, Y. Mitsui, and M. Sugito, "Analysis, synthesis and perception of accent types," Trans. Comm. Speech Res., Acoust. Soc. Jpn. S73-51 (1974) (in Japanese).

5) H. Fujisaki and M. Sugito, "Analysis and perception of two-mora word accent types in the Kinki dialect," J. Acoust. Soc. Jpn. 34, 167-176 (1978) (in Japanese).

6) K. Hirose, H. Fujisaki, and M. Sugito, "Word accent in Japanese and English: A comparative study of acoustic characteristics in disyllabic words," J. Acoust. Soc. Am. 64, Suppl. 1, S114 (1978).

7) H. Fujisaki and H. Sudo, "A model for the generation of fundamental frequency contours of Japanese word accent," J. Acoust. Soc. Jpn. 27, 445-453 (1971) (in Japanese).

8) H. Fujisaki, N. Nakamura, and K. Yoshimune, "Analysis, normalization and recognition of sustained Japanese vowels," J. Acoust. Soc. Jpn. 26, 152-154 (1970). 